\title{
QUALITY OF SERVICE PARAMETERS AND LINK OPERATING POINT ESTIMATION BASED ON EFFECTIVE BANDWIDTHS
}

\author{
$A R T E S^{*}$ \\ FACULTAD DE INGENIERÍA, UNIVERSIDAD DE LA REPÚBLICA \\ MONTEVIDEO, URUGUAY
}

\begin{abstract}
This work addresses the estimation and calculation of the operating point of a network's link in a digital traffic network. The notion of operating point comes from Effective Bandwidth (EB) theory. The results are valid for a wide range of traffic types. This means that the statistical characteristics of the traffic may be very general. We show that, given a good EB estimator, the operating point, i.e. the values of time and space (or multiplexing) parameters in which the EB gives the asymptotic overflow probability, can also be accurately estimated.

Imposing some regularity conditions, a consistent estimator and confidence intervals of the operating point are developed. These conditions are very general, and they are met by commonly used estimators as the averaging estimator presented in [2] or the Markov Fluid model estimator presented in [10].

Using a software package developed by our workgroup that estimates the EB and other relevant parameters from traffic traces, simulation results are compared with the analytical results, showing very good fitting.
\end{abstract}

Keywords. Traffic modelling, traffic estimation, effective bandwidths, QoS, MPLS, operating point estimation.

\section{INTRODUCTION}

The usage of digital networks for carrying variable bit rate (VBR) and real time (RT) or time sensitive services is growing. New control mechanisms and protocols are added to existing data oriented networks to give an appropriate support to such services. The state of the art in traffic engineering is briefly described in section 2 .

The resource sharing in these networks is absolutely needed for an economic usage. This issue leads to the problem of estimating the resources needed for guaranteed VBR communications, which cannot be the peak rate nor the mean rate. Indeed, the mean rate would be a too optimistic estimation, that would cause frequent losses. On the other side, the peak rate would be too pessimistic and would lead to a resource waste.

The effective bandwidth (EB) defined by F. Kelly in [8] is an interesting and realistic measure of channel occupancy. The EB is defined as follows:

$$
\alpha(s, t)=\frac{1}{s t} \log \mathbf{E}\left(e^{s X_{t}}\right) \quad 0<s, t<\infty .
$$

where $X_{t}$ is the total amount of work arriving from a source in the time interval $[0, t]$, which is supposed to be a stochastic process with stationary increments. $\alpha(s, t)$ lies between the mean rate (for $s \rightarrow 0$ ) and the peak rate (for $s \rightarrow \infty$ ) of the input process.

Parameters $s$ and $t$ are referred to as the space and time parameters respectively. When solving for a specific performance guarantee, these parameters depend not only on the source itself, but on the context on which this source is acting. More specifically, $s$ and $t$ depend on the capacity, buffer size and scheduling policy of the multiplexer, the QoS parameter to be achieved, and the

* ARTES: Joint Research Group of the Electrical Engineering and Mathematics and Statistics Departments. Members: Laura Aspirot, Pablo Belzarena, Paola Bermolen, Andrés Ferragut, Gonzalo Perera and María Simon. Contact: artes@fing.edu.uy. 
actual traffic mix (i.e. characteristics and number of other sources). The concept can be applied to sources or to aggregate traffic, as we find in a network's core link.

Under the many sources asymptotic regime discussed in [2], where it is assumed that, as the number of sources feeding a switch grows, the switch capacity and buffer size increase proportionally, the EB is related with the stationary loss probability through buffer overflow by the so called inf sup formula:

$$
\Gamma=\inf _{t \geq 0} \sup _{s \geq 0}((B+C t) s-N s t \alpha(s, t))
$$

where $C$ is the link capacity, $B$ is its buffer capacity and $N$ the number of incoming multiplexed sources of effective bandwidth $\alpha(s, t)$. If $Q_{N}$ represents the stationary amount of work in the queue, the buffer overflow probability or loss probability is approximately given by:

as shown in [2] and [14].

$$
\log \mathbf{P}\left(Q_{N}>B\right) \approx-\Gamma
$$

We call $s^{*}$ and $t^{*}$ the values of parameters $s$ and $t$ in which the inf sup is attained. These values $s^{*}$ and $t^{*}$ are called the operating point of the link.

Therefore, a good estimation of $s^{*}$ and $t^{*}$ is useful for the network's design, for the Connection Acceptance Control (CAC) function, or for optimal operation.

The technical relevance of the issue is pointed out in section 2, where we briefly present the traffic engineering in Multiprotocol Label Switching (MPLS) networks. We point out the need of a good estimation of the bandwidth in order to optimize the resource sharing.

In section 3 we show how the operating point of a link can be estimated from its EB, the consistency of this estimation and its confidence interval. We observe that other well known estimators fit the necessary conditions for the validity of the theorem.

Our workgroup has developed software that estimates the EB from general traffic traces, calculates it when an explicit model is given, estimate the values of $s^{*}$ and $t^{*}$ and deduces then the overflow or loss probability and other relevant design parameters as $B$ (buffer size) and $C$ (link capacity). Using this tool, the EB and operating point of traffic traces were obtained, and their dispersion was estimated. Analytical results are compared with numerical data in section 4. These numerical data were obtained from simulations models that are explained in this section, independently from the analytical work.

In section 5 we present several conclusions and indicate ways for further work.

\section{Motivation}

The convergence of the different telecommunications services on a unique network is an already old aspiration. Integrated Service Digital Network (ISDN) has been a goal for the Telecommunications community from long time ago. General functionalities for broadband networks were defined, as in [5] or [6]. Nevertheless, many candidates have failed to implement such integrated network. However, the research and proposals lead to some basic designs principles and measurement methods that prevail and apply to the new proposals. One of the most important of them, and that is a central point in this work is guarantee a Quality of Service (QoS), and how to achieve it.

Currently, the most promising approach to an integrated service network are the already old IP networks. And maybe the most attractive new service to be given is voice, the most traditional telecommunication service. But in this case, the service and the network come from different worlds. In fact, services as voice are given on IP platforms, but without any guarantee of quality. This is because plain IP networks are based in a "best effort" policy, well suited for data but not for time sensitive applications. In order to support variable bit rate (VBR) and time sensitive services, the concept of QoS must be implemented over IP. Many proposals were done, incorporating some mechanisms, partially inspired in ATM definitions. Multi Protocol Label Switching architecture (MPLS) presented in [12] seems a good working proposal to support a variety of services, including voice, data and video. It can handle VBR streams, and control the path each stream follows. 
However, in order to give a guaranteed QoS, MPLS must be complemented with traffic engineering tools [1]. Its efficiency regarding the usage of the network's capacity depends on the accurate prediction of the bandwidth needs of each flow. Such a prediction can be done for a source (voice, video, data) or for aggregate traffic. It should be accurate enough to give efficiency, and simple enough to be performed in practical conditions.

Our workgroup is working in some issues related with MPLS, because we believe this protocol is a promising way to implement end-to-end QoS over IP. MPLS introduce the notion of Forwarding Equivalence Class (FEC), giving the network operator the possibility to partition the traffic in aggregate flows according to the service model adopted by the Internet Service Provider (ISP).

The edge routers in an MPLS network (or LSR for Label Switch Router) are responsible for establishing MPLS tunnels named LSPs (Label Switched Path) between the endpoints of the communication, and to send each arriving packet to the corresponding LSP.

Explicit Routing in MPLS networks is the main function that enable Traffic Engineering. With explicit routing the network operator can establish for each FEC one or more LSPs. But, given a LSPs configuration defined by the operator: is it possible to ensure the level of service required by each Service Level Agreement (SLA)? And if it is not possible: how can the operator use traffic engineering to give the QoS performance required for each aggregate flow?

The main goal of this work is to give some theoretical insight about the previous questions, and to develop a practical tool for network design and performance optimisation questions. We are especially interested in traffic engineering methodologies based on the statistical characterization of different flows. In this framework the overflow probability estimation is a key topic, and this is what makes necessary the EB and the link operating point estimation.

Depending on the technical possibilities, the results about EB and operating point estimation can be used for off line design, for Connection Acceptance Control (CAC) in the edge LSRs or even for routing in the core LSRs. These applications are successively more demanding in computing capacity of the switches. At present time we focus primarily on the design issues.

Even though this work was motivated by MPLS traffic engineering, its results are useful for a general network that handles VBR communications and has to provide a fixed or at least consistent QoS. It is valid more generally, for problems of limited resource sharing in which some guarantees of loss and delay are intended to be met.

\section{Estimation}

Estimating the operating point of a link as defined in section 1 is closely related with its defining equation which we rewrite here on a per source basis:

$$
\gamma=\inf _{t \geq 0} \sup _{s \geq 0}((b+c t) s-s t \alpha(s, t))
$$

where $\gamma$ is the asymptotic decay rate of the overflow probability as the number of sources increases, $c$ and $b$ are the link's capacity and buffer size per source and $\alpha(s, t)$ the effective bandwidth function of the incoming traffic, also defined in section 1 :

$$
\alpha(s, t)=\frac{1}{s t} \log \mathbf{E}\left(e^{s X_{t}}\right) \quad 0<s, t<\infty .
$$

With the present notation, the stationary overflow probability in a switch multiplexing $N$ sources, having capacity $C=N c$ and buffer size $B=N b$ verifies:

$$
\lim _{N \rightarrow \infty} \frac{1}{N} \log \mathbf{P}\left(Q_{N}>B\right)=-\gamma
$$

In general, the effective bandwidth function $\alpha(s, t)$ is unknown, and shall be estimated from measured traffic traces. The problem is how to estimate the moment generating function $\Lambda(s, t)=$ $\mathbf{E}\left(e^{s X_{t}}\right)$ of the incoming traffic process $X_{t}$ for each $s$ and $t$. 
Different approaches have been presented to solve this problem. One of them, presented in [3] and [11] is to estimate the expectation $\mathbf{E}\left(e^{s X_{t}}\right)$ as the time average given by:

$$
\Lambda_{n}(s, t)=\frac{1}{n} \sum_{k=1}^{n} e^{s\left(X_{k t}-X_{(k-1) t}\right)}
$$

which is valid if the process increments are stationary and satisfy any weak dependence hypothesis that guarantees ergodicity. To estimate $\Lambda(s, t)$ is necessary a traffic trace of length $T=n t$. We can construct an appropriate estimator of the $\mathrm{EB}$ as $\alpha_{n}(s, t)=\frac{1}{s t} \log \left(\Lambda_{n}(s, t)\right)$.

When a model is available for the incoming traffic, a parametric approach can be taken. In the case of a Markov Fluid model, i.e., when the incoming process is modulated by a continuous time Markov chain which dictates the rate of incoming work, explicit computation can be made as shown by Kesidis et. al. in [9]. In this case, an explicit formula is given for $\Lambda(s, t)$ and $\alpha(s, t)$ in terms of the infinitesimal generator or $Q$-matrix of the Markov chain. In a previous work of our group [10], and based on the maximum likelihood estimators of the $Q$-matrix parameters presented in [7], an EB estimator and confidence intervals are developed.

Having an estimator of the function $\alpha(s, t)$, one is tempted to estimate $\gamma$, and the operating point $s^{*}, t^{*}$ substituting the function $\alpha(s, t)$ by $\alpha_{n}(s, t)$ in equation (1) and solving the remaining optimization problem. The output would be some values of $\gamma_{n}, s_{n}^{*}$ and $t_{n}^{*}$, and the question is under what conditions these values are good estimators of the real $\gamma, s^{*}$ and $t^{*}$.

Therefore, we may discuss two different problems concerning estimation. The first one is, given a "good" estimator $\alpha_{n}(s, t)$ of $\alpha(s, t)$, find sufficient conditions under which the estimators $s_{n}^{*}, t_{n}^{*}$ and $\gamma_{n}^{*}$ obtained by solving the optimization problem:

$$
\gamma_{n}=\inf _{t \geq 0} \sup _{s \geq 0}\left((b+c t) s-s t \alpha_{n}(s, t)\right)
$$

are "good" estimators of the operating point $s^{*}, t^{*}$ and the overflow probability decay rate $\gamma$ of a link. This affirmation is not an obvious because $s^{*}$ and $t^{*}$ are found from a non linear and implicit function.

The second problem is finding such good estimators of the EB and determining whether the conditions are met so that the operating point can be estimated using equation (5).

The remaining part of the section addresses the first problem, where a complete answer concerning consistency and Central Limit Theorem (CLT) properties of estimators is given by theorem 1 , based on regularity conditions of the EB function. At the end of the section we discuss the validity of the theorem for some known estimators and in section 4 we compare our answers with numerical results.

Let us define:

$$
g(s, t)=s(b+c t)-s t \alpha(s, t)
$$

which can be rewritten in terms of $\Lambda(s, t)=\mathbf{E}\left(e^{s X_{t}}\right)$ as:

$$
g(s, t)=s(b+c t)-\log (\Lambda(s, t))
$$

Then we have that $\frac{\partial}{\partial s} g(s, t)=0$ if and only if:

$$
\frac{\partial}{\partial s} g(s, t)=b+c t-\frac{\frac{\partial}{\partial s} \Lambda(s, t)}{\Lambda(s, t)}=0
$$

Assume that for each $t$ there exists $s(t)$ such that,

$$
\frac{\partial}{\partial s} g(s(t), t)=0
$$

It is easy to show that $\sup _{s \geq 0} g(s, t)=g(s(t), t)$ because $g(s, t)$ is convex as a function of $s$. In that case, $\gamma=\inf _{t \geq 0} g(s(t), t)$, and:

$$
\frac{\partial}{\partial t}(g(s(t), t))=\frac{\partial}{\partial s} g(s(t), t) \dot{s}(t)+\frac{\partial}{\partial t} g(s(t), t)
$$


where $\frac{\partial}{\partial t} g(s(t), t)$ corresponds to $\left.\frac{\partial}{\partial t} g(s, t)\right|_{s=s(t)}$.

If there exists $t^{*}$ such that:

$$
\frac{\partial}{\partial t} g\left(s\left(t^{*}\right), t^{*}\right)=0
$$

it follows that:

$$
\gamma=g\left(s\left(t^{*}\right), t^{*}\right)
$$

If we define $s^{*}=s\left(t^{*}\right)$, we have that $\gamma=g\left(s^{*}, t^{*}\right)$ where:

$$
\begin{aligned}
\frac{\partial}{\partial s} g\left(s^{*}, t^{*}\right) \dot{s}\left(t^{*}\right)+\frac{\partial}{\partial t} g\left(s^{*}, t^{*}\right) & =0 \\
\frac{\partial}{\partial s} g\left(s^{*}, t^{*}\right) & =0
\end{aligned}
$$

and then we have the relations:

$$
\frac{\partial}{\partial s} g\left(s^{*}, t^{*}\right)=\frac{\partial}{\partial t} g\left(s^{*}, t^{*}\right)=0
$$

Since:

$$
\frac{\partial}{\partial t} g(s, t)=c s-\frac{\frac{\partial}{\partial t} \Lambda(s, t)}{\Lambda(s, t)}
$$

it follows from (6), (7) and (8) that the operating point must satisfy the equations:

$$
\begin{aligned}
b+c t^{*}-\frac{\frac{\partial}{\partial s} \Lambda\left(s^{*}, t^{*}\right)}{\Lambda\left(s^{*}, t^{*}\right)} & =0 \\
c s^{*}-\frac{\frac{\partial}{\partial t} \Lambda\left(s^{*}, t^{*}\right)}{\Lambda\left(s^{*}, t^{*}\right)} & =0
\end{aligned}
$$

If we make the additional assumptions that interchanging the order of the differential and expectation operators is valid, and that $\dot{X}_{t}$ exists for almost every $t$ we can write:

$$
\frac{\partial}{\partial s} \Lambda(s, t)=\mathbf{E}\left(X_{t} e^{s X_{t}}\right) \quad \frac{\partial}{\partial t} \Lambda(s, t)=\mathbf{E}\left(s \dot{X}_{t} e^{s X_{t}}\right)
$$

Replacing the expressions of (10) in equations (9) we deduce an alternative expression for the solutions $s^{*}$ and $t^{*}$ :

$$
\begin{aligned}
& b+c t^{*}-\frac{\mathbf{E}\left(X_{t^{*}} e^{s^{*} X_{t^{*}}}\right)}{\mathbf{E}\left(e^{s^{*} X_{t^{*}}}\right)}=0 \\
& c s^{*}-\frac{\mathbf{E}\left(s^{*} \dot{X}_{t^{*}} e^{s^{*} X_{t^{*}}}\right)}{\mathbf{E}\left(e^{s^{*} X_{t^{*}}}\right)}=0
\end{aligned}
$$

Therefore, we can reformulate the optimization problem presented in (1). The operating point of the link can be calculated solving the system of equations (9), or (11) if the additional assumptions are valid. The first formulation, which is more general, is the one used in the main result of this work, which follows:

Theorem 1. If $\Lambda_{n}(s, t)$ is an estimator of $\Lambda(s, t)$ such that both are $C^{1}$ functions and:

$$
\begin{aligned}
\Lambda_{n}(s, t) & \longrightarrow \\
\frac{\partial}{\partial s} \Lambda_{n}(s, t) & \longrightarrow(s, t) \\
\frac{\partial}{\partial t} \Lambda_{n}(s, t) & \frac{\partial}{\partial s} \Lambda(s, t) \\
& \frac{\partial}{\partial t} \Lambda(s, t)
\end{aligned}
$$


almost surely and uniformly over bounded intervals, and if we denote $s_{n}^{*}$ and $t_{n}^{*}$ the solutions of:

$$
\begin{aligned}
b+c t_{n}^{*}-\frac{\frac{\partial}{\partial s} \Lambda_{n}\left(s_{n}^{*}, t_{n}^{*}\right)}{\Lambda_{n}\left(s_{n}^{*}, t_{n}^{*}\right)} & =0 \\
c s_{n}^{*}-\frac{\frac{\partial}{\partial t} \Lambda_{n}\left(s_{n}^{*}, t_{n}^{*}\right)}{\Lambda_{n}\left(s_{n}^{*}, t_{n}^{*}\right)} & =0
\end{aligned}
$$

then $\left(s_{n}^{*}, t_{n}^{*}\right)$ are consistent estimators of $\left(s^{*}, t^{*}\right)$. Moreover, if a functional Central Limit Theorem (CLT) applies to $\Lambda_{n}-\Lambda$, i.e,

$$
\sqrt{n}\left(\Lambda_{n}(s, t)-\Lambda(s, t)\right) \stackrel{w}{\underset{n}{\longrightarrow}} G(s, t),
$$

where $G(s, t)$ is a continuous gaussian process, then:

$$
\sqrt{n}\left(\left(s_{n}^{*}, t_{n}^{*}\right)-(s, t)\right) \stackrel{w}{\Longrightarrow} N(\overrightarrow{0}, \Sigma)
$$

where $N(\overrightarrow{0}, \Sigma)$ is a centered bivariate normal distribution with covariance matrix $\Sigma$.

Proof. From equations (9), we know that $\left(s^{*}, t^{*}\right)$ is the solution of $K((s, t), \Lambda)=\overrightarrow{0}$ where:

$$
K((s, t), \Lambda)=\left(\begin{array}{c}
b+c t-\frac{\frac{\partial}{\partial s} \Lambda(s, t)}{\Lambda(s, t)} \\
c s-\frac{\frac{\partial}{\partial t} \Lambda(s, t)}{\Lambda(s, t)}
\end{array}\right)
$$

and $\left(s_{n}^{*}, t_{n}^{*}\right)$ is the solution of $K\left(\left(s_{n}, t_{n}\right), \Lambda_{n}\right)=\overrightarrow{0}$.

Recall that if $E, F$ are normed spaces (or more in general semi-normed spaces), $f: E \rightarrow F$ is said to be differentiable at $e \in E$ if there exists a continuous linear map $d f(e): E \rightarrow F$ such that for any $e^{\prime}$ in a neighborhood at $e$ we have that

$$
f\left(e^{\prime}\right)=f(e)+d f(e)\left(e^{\prime}-e\right)+o\left(\left\|e^{\prime}-e\right\|\right)
$$

Let also recall that if $E_{1}, E_{2}, F$ are normed spaces and $f: E_{1} \times E_{2} \rightarrow F$ is differentiable so are, for any $e_{1} \in E_{1}$ and $e_{2} \in E_{2}, f_{e_{1}}: E_{2} \rightarrow F$ defined by $f_{e_{1}}\left(e_{2}\right)=f\left(e_{1}, e_{2}\right)$ end $f^{e_{2}}: E_{1} \rightarrow F$ defined by $f^{e_{2}}\left(e_{1}\right)=f\left(e_{1}, e_{2}\right)$.

Finally, let us recall the general form of the implicit function derivative formula. If $f$ is differentiable, and for each $e_{2} \in E_{2}$ there exists an unique element $v\left(e_{2}\right)$ of $E_{1}$ such that $f\left(v\left(e_{2}\right), e_{2}\right)=\overrightarrow{0}$ and $d f^{e_{2}}\left(v\left(e_{2}\right)\right)$ is invertible, then

$$
\begin{gathered}
d f^{e_{2}}\left(v\left(e_{2}\right)\right) d v\left(e_{2}\right)+d f_{v\left(e_{2}\right)}\left(e_{2}\right)=0, \\
d v\left(e_{2}\right)=-\left(d f^{e_{2}}\left(v\left(e_{2}\right)\right)\right)^{-1} d f_{v\left(e_{2}\right)}\left(e_{2}\right)
\end{gathered}
$$

We will apply (17) to $E_{1}=\left(\mathbb{R}^{+}\right)^{2}$ equipped with the euclidean norm and

$$
E_{2}=\left\{f:\left(\mathbb{R}^{+}\right)^{2} \rightarrow \mathbb{R} \text { of class } C^{1}\right\}
$$

equipped with the seminorm

$$
\|f\|=\sum_{n=1}^{\infty} \frac{1}{2^{n}}\left(\frac{\|f\|_{n}}{1+\|f\|_{n}}\right)
$$

where $\|f\|_{n}$ is the Sobolev-type norm

$$
\sup \left\{|f(s, t)|+\left|\frac{\partial}{\partial s} f(s, t)\right|+\left|\frac{\partial}{\partial t} f(s, t)\right|:(s, t) \in[0, n]^{2}\right\}
$$

It is easy to check that, for a sequence $\left\{f_{m}\right\}$ of functions of $E_{2},\left\|f_{m}\right\| \rightarrow 0$ when $m \rightarrow \infty$ if and only if $f_{m} \rightarrow 0, \frac{\partial}{\partial s} f_{m} \rightarrow 0, \frac{\partial}{\partial t} f_{m} \rightarrow 0$ uniformly over bounded intervals when $m \rightarrow \infty$.

Applying the preceding paragraph, we may think of $\left(s^{*}, t^{*}\right)$ as $(s, t)(\Lambda)=v(\Lambda)$ and by $(17)$ :

$$
d v(\Lambda)=-\left(d K^{\Lambda}(v(\Lambda))\right)^{-1} d K_{v(\Lambda)}(\Lambda)
$$


Therefore, since $\left(s_{n}^{*}, t_{n}^{*}\right)=v\left(\Lambda_{n}\right)$ :

$$
\left(s_{n}^{*}, t_{n}^{*}\right)-(s, t)=v\left(\Lambda_{n}\right)-v(\Lambda)=d v(\Lambda)\left(\Lambda_{n}-\Lambda\right)+o\left(\left\|\Lambda_{n}-\Lambda\right\|\right)
$$

But the hypotheses of the theorem imply that $\left\|\Lambda_{n}-\Lambda\right\| \underset{n}{\longrightarrow} 0$ a.s and $d v(\Lambda)$ is continuous, therefore:

$$
\left(s_{n}^{*}, t_{n}^{*}\right) \underset{n}{\longrightarrow}\left(s^{*}, t^{*}\right) \text { a.s. }
$$

showing consistency.

If in addition, a functional CLT applies to $\Lambda_{n}-\Lambda$, i.e,

$$
\sqrt{n}\left(\Lambda_{n}(s, t)-\Lambda(s, t)\right) \stackrel{w}{\underset{n}{\longrightarrow}} G(s, t),
$$

where $G(s, t)$ is a $C^{1}$ gaussian process, we have that

$$
\sqrt{n}\left(\left(s_{n}^{*}, t_{n}^{*}\right)-(s, t)\right)=d v(\Lambda)\left(\sqrt{n}\left(\Lambda_{n}-\Lambda\right)\right)+o\left(\sqrt{n}\left\|\Lambda_{n}-\Lambda\right\|\right)
$$

Since $\sqrt{n}\left\|\Lambda_{n}-\Lambda\right\| \stackrel{w}{\underset{n}{\rightleftharpoons}}\|G\|$, it is bounded in probability and, therefore,

$$
o\left(\sqrt{n}\left\|\Lambda_{n}-\Lambda\right\|\right) \underset{n}{\stackrel{(p)}{\longrightarrow}} 0
$$

On the other hand, since $d v(\Lambda)$ is continuous, we have that

$$
\sqrt{n} d v(\Lambda)\left(\Lambda_{n}-\Lambda\right) \stackrel{w}{\underset{n}{\longrightarrow}} d v(\Lambda)(G)
$$

Therefore, we have finally shown that:

$$
\sqrt{n}\left(\left(s_{n}^{*}, t_{n}^{*}\right)-(s, t)\right) \stackrel{w}{\underset{n}{\longrightarrow}} d v(\Lambda)(G)
$$

which, being $d v(\Lambda)$ a linear transformation into $\mathbb{R}^{2}$, implies that $d v(\Lambda)(G)$ is a normal $N(\overrightarrow{0}, \Sigma)$ bivariate random variable, where $\Sigma$ may be computed in terms of the covariances of $G$ and the transformation $d v(\Lambda)$.

Remark. As can be seen from the proof, computation of $\Sigma$ is not trivial. But the result of normality for the estimation allows, if replication is possible (for instance by taking large traces of weakdependent signals), the estimation of $\Sigma$ in terms of empirical covariances. Arguments of this type are used in section 4 .

Remark. Since the convergence assured by theorem 1 is uniform over bounded intervals, it is also assured that $\gamma_{n}$ given by:

$$
\gamma_{n}=s_{n}^{*}\left(b+c t_{n}^{*}\right)-\Lambda_{n}\left(s_{n}^{*}, t_{n}^{*}\right)
$$

inherits the properties of the $s_{n}^{*}$ and $t_{n}^{*}$ estimators. That is, $\gamma=F\left(s^{*}, t^{*}, \Lambda\right)$ where $F$ is a differentiable function. Also, $\gamma_{n}=F\left(s_{n}^{*}, t_{n}^{*}, \Lambda_{n}\right)$. Therefore, if the estimator $\Lambda_{n}$ verifies a functional CLT we have for $\gamma_{n}$ :

$$
\sqrt{n}\left(\gamma_{n}-\gamma\right) \stackrel{w}{\Longrightarrow} N\left(0, \sigma^{2}\right)
$$

Remark. In a many source environment as will be envisaged in section 4 expressions for the buffer size $b$ and the link capacity $c$ obtained by Courcoubetis [3] are similar to the inf sup equation. Therefore, using the reasoning used in the previous theorem extends consistency and CLT results to $b^{*}$ and $c^{*}$. Therefore, confidence intervals for design parameters can be constructed in this way.

We address now the second question posed at the beginning of the section. As we can see, for the validity of theorem 1 it is necessary that the estimator $\Lambda_{n}(s, t)$ converge uniformly to the moment generating function over bounded intervals, as well as its partial derivatives. These conditions are reasonably general, and it can be verified that they are met by the estimator (4) presented in [3] and [11], and by the estimator for Markov Fluid sources presented in [10]. In both cases a CLT can be obtained so the CLT conclusion of the theorem is also valid. It should be noticed that a consistent but non-smooth estimator can be used with this procedure, if it is previously regularized by convolution with a suitable kernel. 


\section{Simulation AND NUMERICAL RESUlts}

4.1. Introduction. In the analysis of networks performance using Large deviations theory [4] two asymptotic regimes have been described. These are the large buffer regime and the many sources regime.In the first case the convergence rate to zero of some QoS parameter (for example, loss probability) when the buffer size goes to infinity is studied. In the second one it is also studied the convergence rate to zero of the loss probability but when there ar many independent and identically distributed sources arriving at the link and the link capacity and the buffer size both increases at the same rate as the number of sources.

This work will be focused in networks such as an internet backbone, where powerful design and analysis tools are needed. In those networks the many sources asymptotic approach is more reasonable than the large buffer one. In a backbone of this kind a large numbers of flows from different sources arrives, there are important capacities and the buffer size per source are in general small, because they are thought to serve many sources but not many bursts at the same time. If the network is designed to serve many bursts at the same time it would carry out very large buffers. On the other hand, for many sources with statistical multiplexing, aggregate flows are expected to have less bursts than individual ones. That is why the large buffer regime is less useful than the many sources regime in these networks.

In the many sources regime the loss probability could be obtained from the solution of the inf sup formulae (1). To solve this equation a double optimization (in time and space parameters) is needed, in order to obtain the link operating point $\left(s^{*}, t^{*}\right)$. The first problem is that in real cases, when is not assumed a model for the source, the is not an explicit formulae for the EB. In the general case the information available is about traces, and the equation (1) must be solved in terms of $\alpha_{n}(s, t)$ (an EB estimator) instead of $\alpha(s, t)$. From previous sections we know that the link operating point estimation obtained from a good estimation $\alpha_{n}(s, t)$ is consistent and has CLT properties. In this work we make the analysis with simulated traces from a known theoretical model in order to evaluate our results. In the next section we explain the model and the EB estimation. After that an estimator of the link operating point will be obtained, and it will be used to calculate the QoS performance parameters and some link design parameters.

4.2. EB estimation. To validate the results obtained in the previous section, we simulated traffic using a two state (ON-OFF) Markov Fluid model. In that model, a continuous time Markov chain drives the process. When the chain is in the ON state, the workload is produced at constant rate $h_{0}$, and when it is in the OFF state no workload is produced $\left(h_{1}=0\right)$. Denoting by $Q$ the infinitesimal generator of the Markov chain, by $\vec{\pi}$ its invariant distribution, and by $H$ the diagonal matrix with the rates $h_{i}$ in the diagonal, the effective bandwidth for a source of this type is [9][8]:

$$
\alpha(s, t)=\frac{1}{s t} \log \{\vec{\pi} \exp [(Q+H s) t] \overrightarrow{\mathbf{1}}\}
$$

where $\overrightarrow{\mathbf{1}}$ is a column vector of ones.

In our simulations we generated three hundred traffic traces of length $T$ samples, with the following $Q$-matrix:

$$
Q=\left(\begin{array}{cc}
-0.02 & 0.02 \\
0.1 & -0.1
\end{array}\right)
$$

The effective bandwidth for this process calculated through equation (19) is shown in figure 1.

For each traffic trace we estimated the effective bandwidth using the following procedure. We divided the trace in blocks of length $t$ and constructed the following sequence:

$$
\tilde{X}_{k}=\sum_{i=(k-1) t}^{k t} x(i) \quad 1 \leq k \leq\lfloor T / t\rfloor
$$

where $x(i)$ is the amount of traffic arrived between samples and $\lfloor c\rfloor$ denotes the largest integer less than or equal to $c$. 


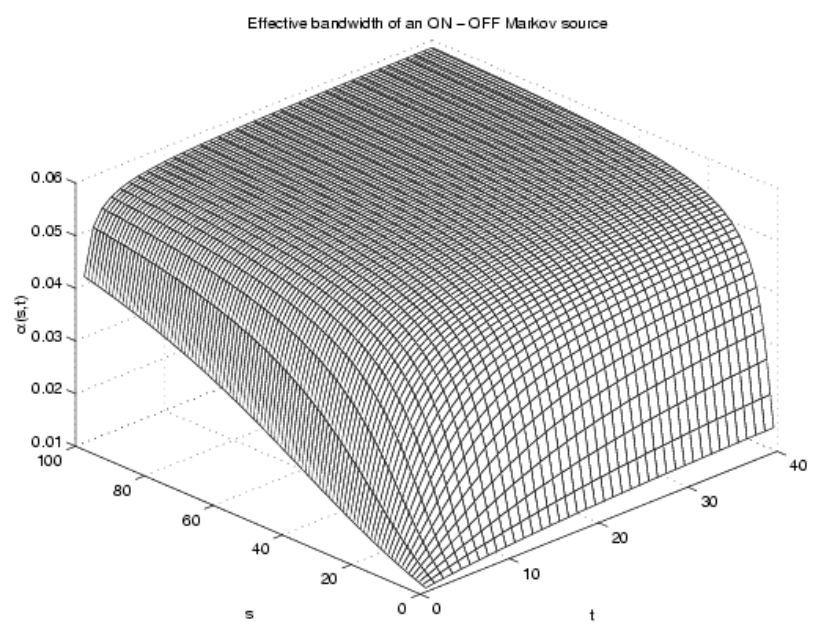

Figure 1. Effective Bandwidth of a Markov Fluid source

The effective bandwidth can then be estimated by the time average proposed in [3], [11] as

$$
\alpha_{n}(s, t)=\frac{1}{s t} \log \left[\frac{1}{\lfloor T / t\rfloor} \sum_{j=1}^{\lfloor T / t\rfloor} e^{s \tilde{X}_{j}}\right]
$$

where $n=\lfloor T / t\rfloor$. This is merely an implementation of the time average estimator in equation (4) based on a finite length traffic trace. When the values of $t$ verify that $t \ll T$, the number of replications of the increment process within the trace is good enough to get a good estimation.

In order to find the operating point $\left(s^{*}, t^{*}\right)$ of the theoretical Markov model, and its estimator $\left(s_{n}^{*}, t_{n}^{*}\right)$ for each simulated trace, we solve the inf sup optimization problem of equation (1). In our case $\alpha(s, t)$ will be the previous theoretical equation (19) for the Markovian source or the $\alpha_{n}(s, t)$ estimated for each trace. The numerical solution has two parts. First, for a fixed $t$ we find the $s^{*}(t)$ that maximize $g(s, t)$ as a function of $s$. It can be shown that $s t \alpha(s, t)$ is a convex function of $s$. This convexity property is used to solve the previous optimization problem, that is reduced to find the maximum difference between a convex function and a linear function of $s$, and it can be done very efficiently. After the $s^{*}(t)$ is found for each $t$, it is necessary to minimize the function $g\left(s^{*}(t), t\right)$ and find $t^{*}$. For this second optimization problem there are no general properties that let us make the search algorithm efficient and a linear searching strategy is used.

The main purpose of our work is to develop a confidence region for $\left(s^{*}, t^{*}\right)$. We simulated $K=$ 300 traces of length $T=100000$ samples and constructed, for each simulated trace indexed by $i=$ $1, \ldots, K$ the corresponding estimator $\left(s_{n}^{*}(i), t_{n}^{*}(i)\right)$. By theorem 1 the vector $\sqrt{n}\left(\left(s_{n}^{*}, t_{n}^{*}\right)-\left(s^{*}, t^{*}\right)\right)$ is asymptotically bivariate normal with mean $(0,0)$ and covariance matrix $\Sigma$. We estimated the matrix $\Sigma$ using the empirical covariances of the observations

$$
\left\{\sqrt{n}\left(\left(s_{n}^{*}(i), t_{n}^{*}(i)\right)-\left(s^{*}(i), t^{*}(i)\right)\right)\right\}_{i=1, \ldots, K}
$$

given by:

$$
\Sigma_{K}=\frac{n}{K}\left(\begin{array}{cc}
\sum_{i=1}^{K}\left(s_{n}^{*}(i)-\overline{s_{n}^{*}}\right)^{2} & \sum_{i=1}^{K}\left(s_{n}^{*}(i)-\overline{s_{n}^{*}}\right)\left(t_{n}^{*}(i)-\overline{t_{n}^{*}}\right) \\
\sum_{i=1}^{K}\left(s_{n}^{*}(i)-\overline{s_{n}^{*}}\right)\left(t_{n}^{*}(i)-\overline{t_{n}^{*}}\right) & \sum_{i=1}^{K}\left(t_{n}^{*}(i)-\overline{t_{n}^{*}}\right)^{2}
\end{array}\right)
$$

where $\overline{s_{n}^{*}}=\frac{1}{K} \sum_{i=1}^{K} s_{n}^{*}(i)$ and $\overline{t_{n}^{*}}=\frac{1}{K} \sum_{i=1}^{K} t_{n}^{*}(i)$.

Therefore, we can say that approximately:

$$
\left(s_{n}^{*}, t_{n}^{*}\right) \approx N\left(\left(s^{*}, t^{*}\right), \frac{1}{n} \Sigma_{K}\right)
$$


from where a level $\alpha$ confidence region can be obtained as:

$$
R_{\alpha}=\left(s_{n}^{*}, t_{n}^{*}\right)+\frac{A_{K}^{t} B\left(\overrightarrow{0}, \sqrt{\chi_{\alpha}^{2}(2)}\right)}{\sqrt{n}}
$$

being $A_{K}$ the matrix that verifies $A_{K}^{t} A_{K}=\Sigma_{K}$ and $B(x, r)$ is the ball of center $x$ and radius $r$.

To verify our results, we calculated the theoretical operating point $\left(s^{*}, t^{*}\right)$ and simulated another 300 traces independent of the used to estimate $\Sigma_{K}$. We constructed then the $95 \%$ confidence region. If the results are right, approximately $95 \%$ of the times, $\left(s^{*}, t^{*}\right)$ must fall inside that region, or equivalently and easier to check, approximately $95 \%$ of the simulated $\left(s_{n}^{*}, t_{n}^{*}\right)$ must fall inside the region $R=\left(s^{*}, t^{*}\right)+\frac{1}{\sqrt{n}} A_{K}^{t} B\left(\overrightarrow{0}, \sqrt{\chi_{0.05}^{2}(2)}\right)$. Numerical results, plotted in figure 2, verify that the confidence level is attained, falling $95.33 \%$ of the estimated inside the predicted region.

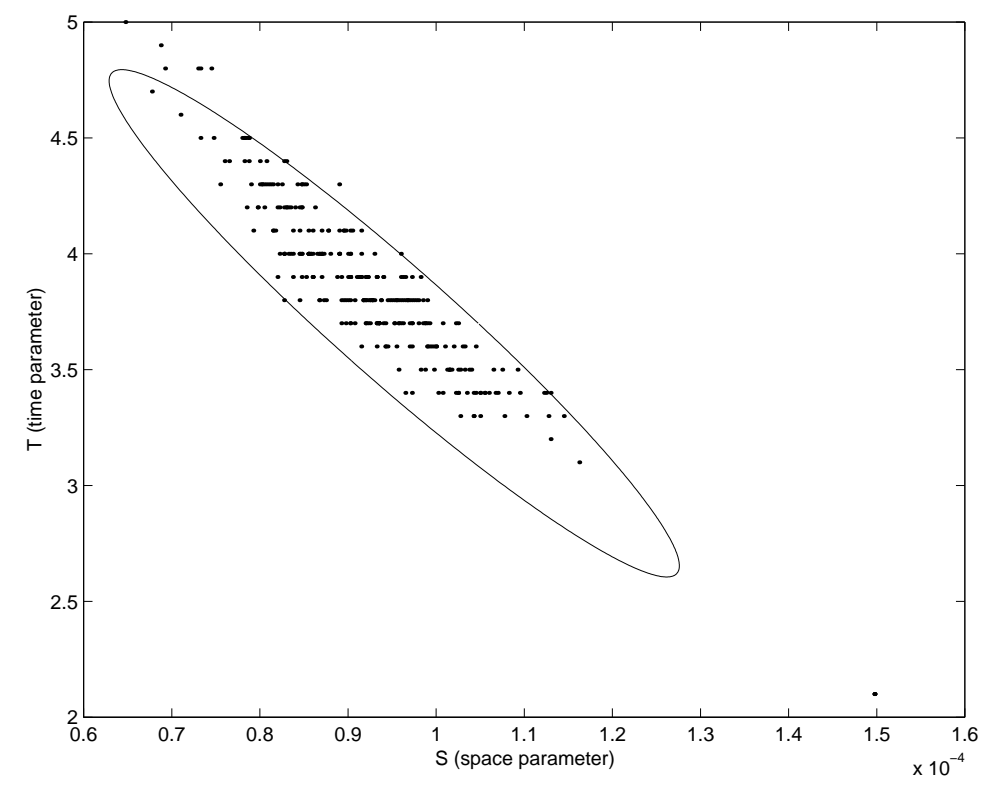

FiGURE 2. Estimated operating points and confidence region

4.3. QoS parameters estimation. We estimate the link operating point in order to estimate loss probability and other QoS parameters, such as delay. In the many sources asymptotic regime, the real delay of packets that cross a link coincides with its virtual delay [13]. The virtual delay is the one obtained through the queue size. If the link sends $C$ packets per unit of time and the probability of having a queue size larger than $B$ is $q$, then the probability of having a delay higher than $B / C$ will be $q$. In this regime if we have an estimator of the probability of having a queue size larger than $B$, we have an estimator of the real delay. We will focus on the estimation of loss probability, because the delay could be deduced from the same equation. As it is said in section 3 , if we have an EB estimator that verifies the hypotheses of theorem 1, then the estimator

$$
\gamma_{n}=\inf _{t} \sup _{s}\left((b+c t) s-s t \alpha_{n}(s, t)\right)
$$

is consistent and has CLT properties. From this estimator the loss probability could be approximated by

$$
q_{n}=P_{n}\left(Q_{N}>B\right) \approx \exp ^{-N \gamma_{n}}
$$

where $Q_{N}$ is the queue size and $N$ is the number of sources. In figure 3 it is shown the estimations of $\gamma_{n}$ for 600 simulated traces, its theoretical value and its confidence interval. Numerical results show that in this case $94.8 \%$ of the values fall in the $95 \%$ confidence interval. 


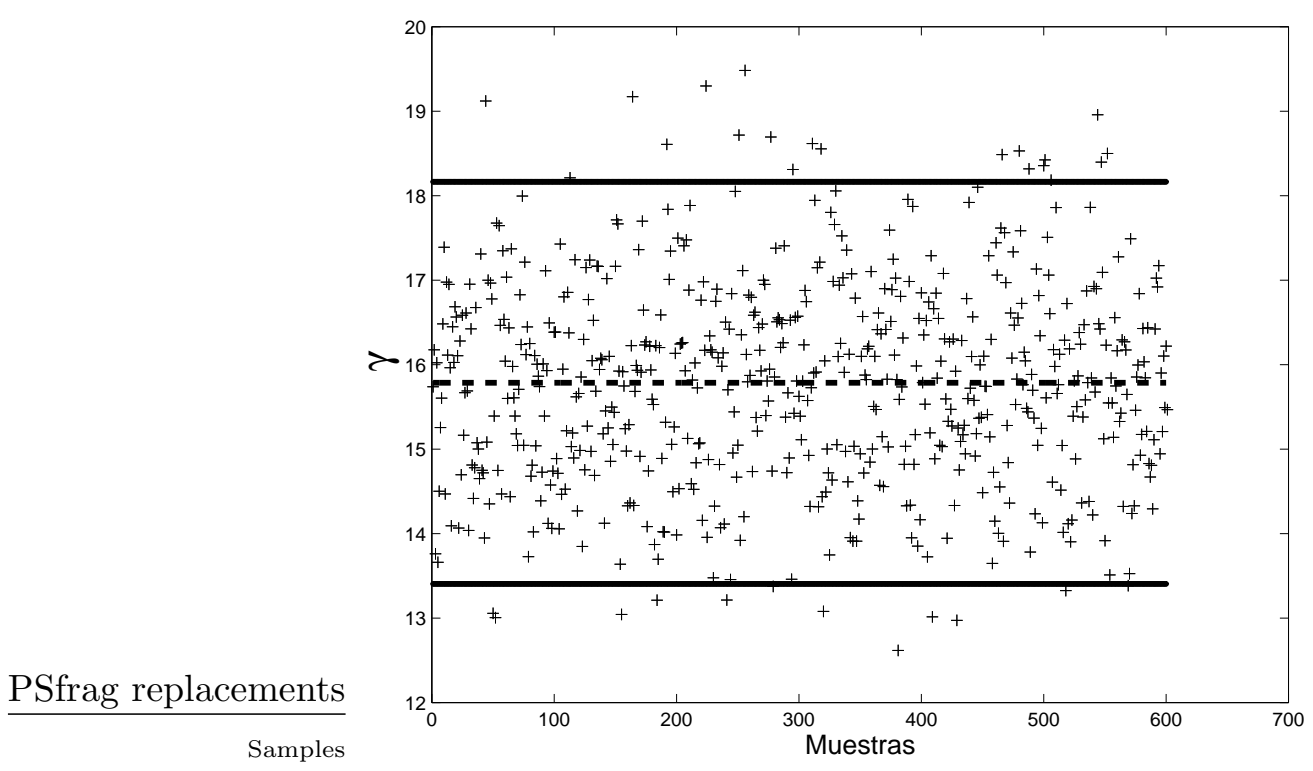

FiguRE 3. Estimation of $\gamma_{n}$, theoretic $\gamma$ and confidence interval.

4.4. Link design based on EB estimation. Previous results could be extended to link design, when some QoS requirements are given. The goal is to know, for a certain link, the smallest buffer size when the capacity $C$, the traffic arriving and the largest loss probability desired (or the largest delay) are given. The same reasoning could be done in order to calculate the smallest necessary link capacity to guarantee the desired loss probability when the same information as before is available but the buffer size is fixed. The answers to these design problems are obtained from equations such as the inf sup formulae. The smallest buffer size to guarantee loss probability $\gamma$ is given [3] by the following equation:

$$
\begin{gathered}
B_{n}=\sup _{t} \inf _{s}\left(G_{n}(s, t)\right) \\
G_{n}(s, t)=\frac{\left(N s t \alpha_{n}(s, t)+N \gamma\right)}{s}-C t
\end{gathered}
$$

and the smallest capacity to guarantee loss probability $\gamma$ is:

$$
\begin{gathered}
C_{n}=\operatorname{supinf}_{t}\left(K_{n}(s, t)\right) \\
K_{n}(s, t)=\frac{\left(N s t \alpha_{n}(s, t)+N \gamma\right)}{s t}-B / t
\end{gathered}
$$

In figures 4 and 5 smallest capacity and buffer size estimations are shown. For each one of the 600 simulated traces $B$ and $C$ have been estimated using the previous equations. In the figures are also indicated the theoretical values and the confidence intervals.

Numerical results for the capacity verify that the confidence level is attained, falling $95 \%$ of the estimated inside the predicted interval. Negative values of buffer size for some traces only show that no buffer is needed to satisfy the desired QoS requirements.

Notice that buffer size has important variation. This fact is related with the operating point in which is the link under design. In figure 6 we plot $-\gamma$ versus buffer size. This is a typical curve of a link where two zones are distinguishable. The first one is for low buffer sizes, where small changes in buffer size lead to important changes in loss probability and the second one shows that to have important changes in loss probability large changes in buffer size are needed. In this case the link is operating in the second zone. Solid and point curves correspond to theoretical $\alpha$ and 


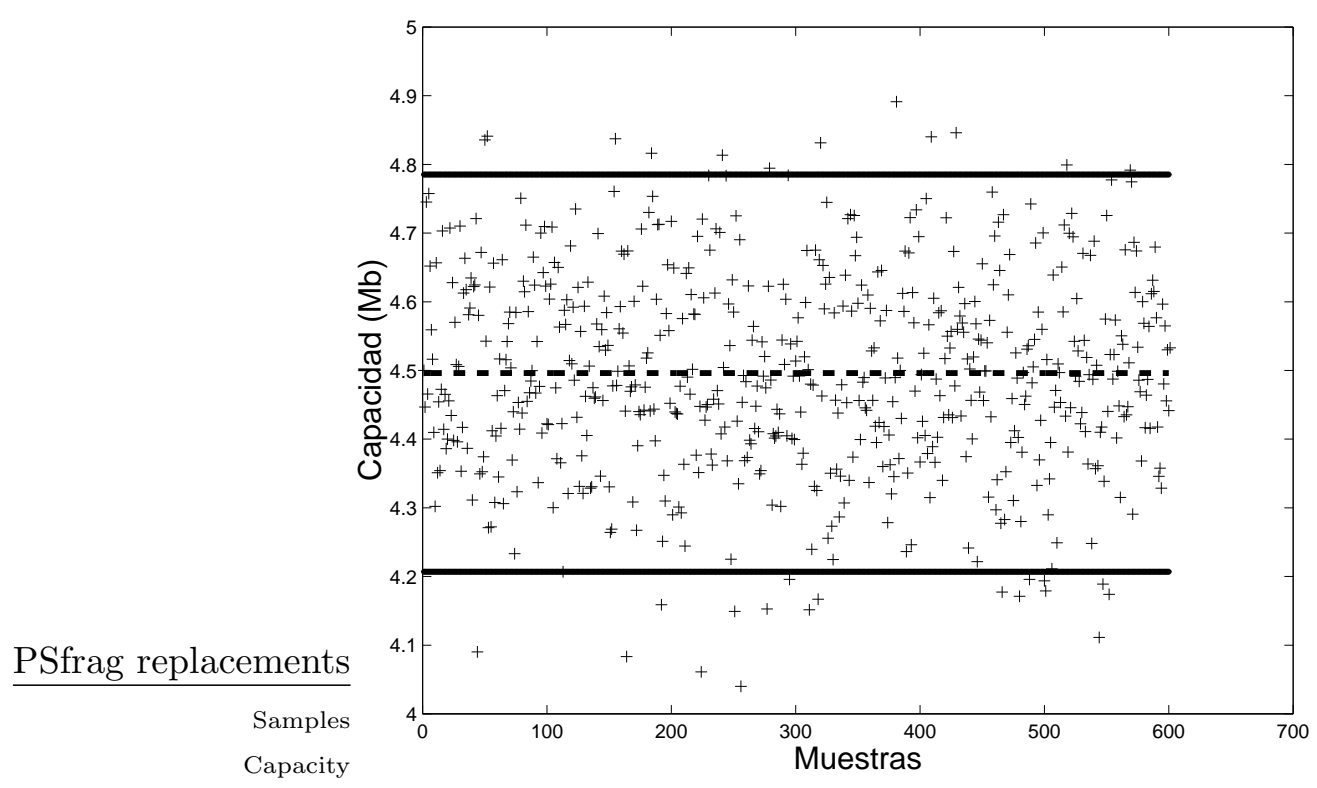

FIGURE 4. Estimated capacity, theoretical capacity and confidence interval.
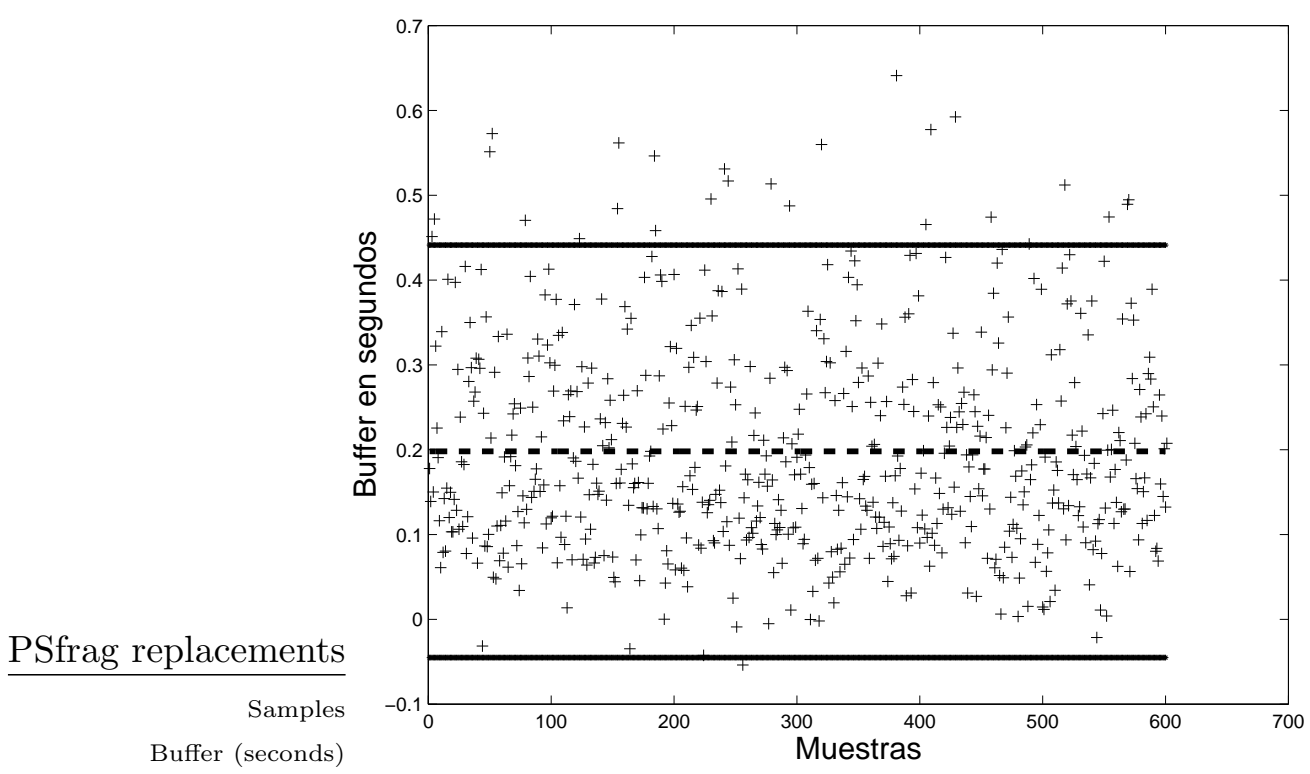

FiguRE 5. Estimated buffer size, theoretical buffer size and confidence interval.

estimated $\alpha_{n}$ respectively. With fixed $B, \gamma$ has little variation when we move from one of these curves to the other. However, if $\gamma$ is fixed $B$ has large variation.

The variation of $\gamma$ with the link capacity $C$ can be studied as before. In figure 7 this variation is shown. The curve slope grows rapidly as $C$ increases, and loss probability goes to zero (so $-\gamma \rightarrow-\infty)$ when $C$ goes to the peak rate. In this case the link is working with capacity equals to $75 \%$ of the peak rate. $\gamma$ has little variation when $C$ is fixed. In the other side, if $\gamma$ is fixed, $C$ has little variation. This fact explains the small variation of $C$ in figure 4.

Our workgroup has developed a software that estimates the effective bandwidth of a source from traffic traces by means of different estimators and finds the operating point, as well as different 


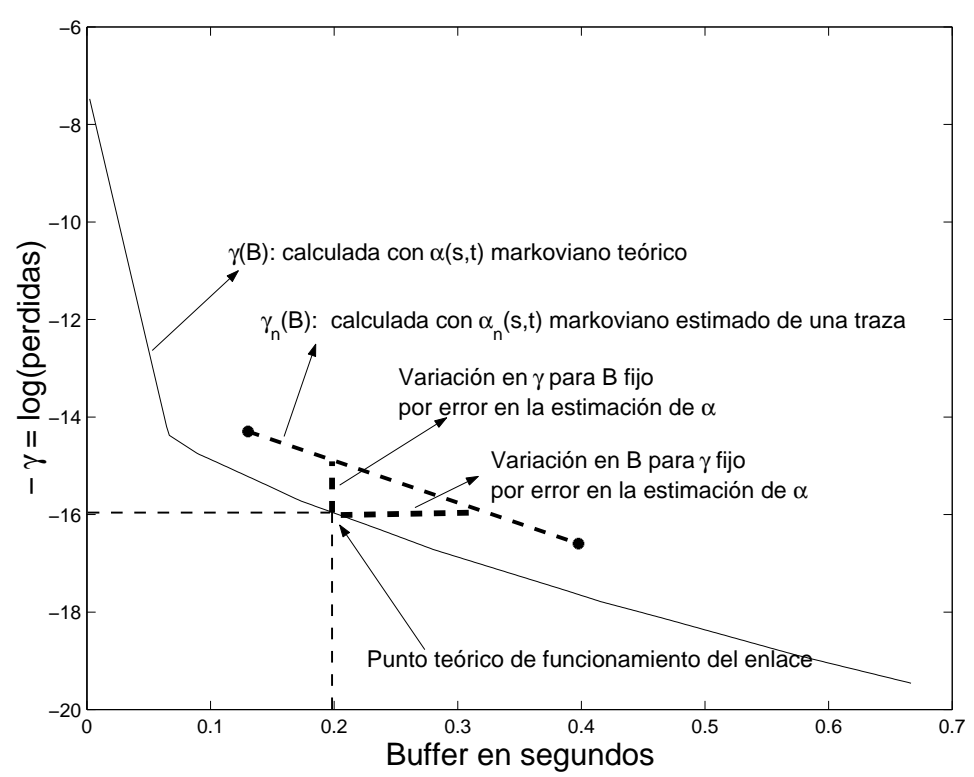

FiguRE $6 .-\gamma$ variation versus buffer size

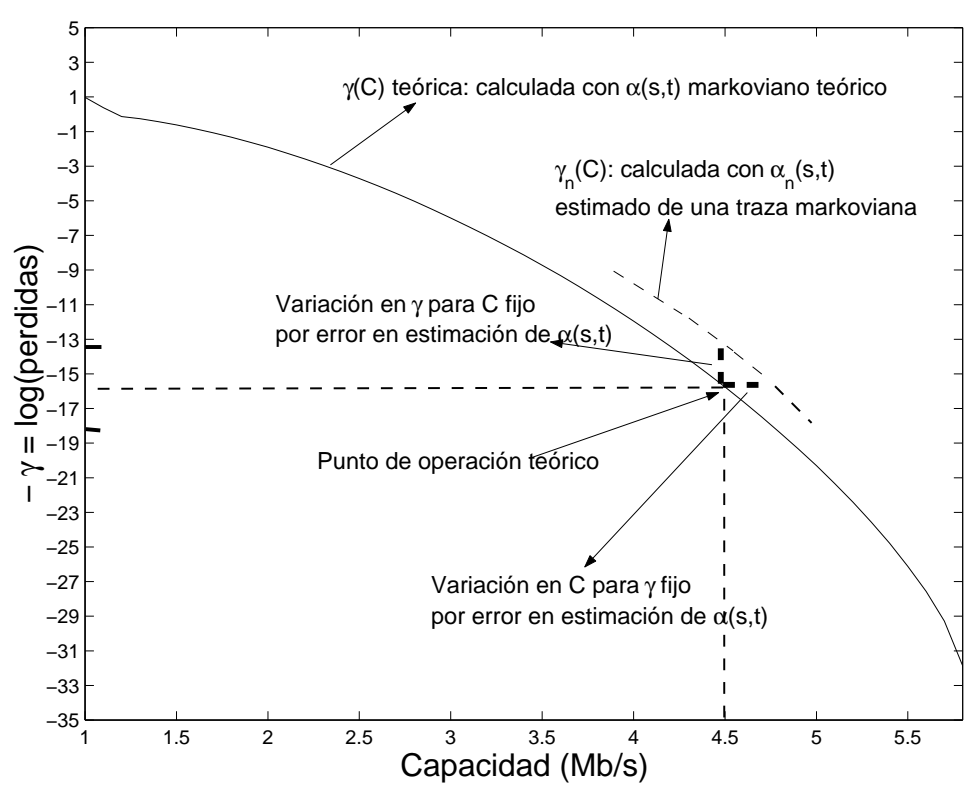

FIGURE 7. $-\gamma$ variation versus link capacity

QoS parameter of a link using our previous framework. The Java source code of the software is available upon request to the authors.

\section{Conclusions}

We have shown that consistency and CLT properties of effective bandwidth estimators can be extended to the operating point estimation through a natural procedure under very general hypothesis. Estimations of this kind are necessary in the analysis and design of networks that must guarantee some degree of QoS to the traffic they carry, in order to support time sensitive services, and in order to optimize the resource usage. This, in turn, is a central problem in the developing of modern networks, where convergence of different services is a goal. 
We have also checked that the numerical estimations constructed by simulation fit very well with the theoretical predictions.

Moreover, the same kind of asymptotic relations that are dealt here with, appear in the estimation of some other QoS parameters, besides the loss probability. In particular we analyze minimum buffer size and capacity estimation that guarantee link operating with a desired loss probability.

We are also working in the extension of this results, as well as the software tools, to the case of a complete network.

\section{REFERENCES}

[1] Awduche, D.; Malcolm, J. (1999), "Requirements for Traffic Engineering Over MPLS", Internet Engineering Task Force RFC 2702.

[2] Courcoubetis, C.; Weber. R (1996), "Buffer overflow asymptotics for a switch handling many traffic sources", Journal of Applied Probability No. 33.

[3] Courcoubetis, C.; Siris, V.A. (2001), "Procedures and tools for analysis of network traffic measurements", Elsevier Science.

[4] Dembo, A.; Zeitouni, O (1993), Large Deviations Techniques and its Applications, Jones and Bartlett, New York.

[5] ITU Recommendation I.211, "General Service Aspects of B-ISDN". ITU-T, Geneva.

[6] ITU Recommendation I.311, "B-ISDN General Network Aspects". ITU-T, Geneva.

[7] Lebedev, E.A.; Lukashuk, L.I. (1986), "Maximum likelihood estimation of the infinitesimal matrix of a Markov chain with continuous time". (Russian, English summary). Dokl. Akad. Nauk Ukr. SSR, Ser. A, No.1, pp. 12-14.

[8] Kelly, F. (1996) "Notes on Effective Bandwidth", in Stochastic Networks: Theory and Applications, edited by Kelly, Zachary and Ziedins, Oxford University Press.

[9] Kesidis, George; Walrand, Jean; Chang, Cheng-Shang (1993), "Effective Bandwidths for Multiclass Markov Fluids and Other ATM Sources", IEEE/ACM Trans. Networking, No.1, pp. 424-428.

[10] Pechiar, J.; Perera, G.; Simon, M. (2002), "Effective Bandwidth estimation and testing for Markov sources", in Performance Evaluation edited by D.D.Kouvatsos, Elsevier, New Holland.

[11] Rabinovitch, P. (2000) "Statistical estimation of effective bandwidth", M.Sc.thesis, University of Cambridge.

[12] Rosen, E.; Viswanathan, A. (2001), "Multiprotocol Label Switching Architecture", Internet Engineering Task Force RFC 3031.

[13] Shakkottai,S.; Srikant, R., "Many-sources Delay Asymptotics with Applications to Priority Queues", Queueing Systems Theory and Applications (QUESTA), Vol. 39, pp. 183-200, October, 2001.

[14] Wischik, D. (2000) "Sample path large deviations for queues with many inputs", Annals of Applied Probability, No. 11, pp. 389-404.

ARtes (Análisis de Redes, Tráfico y Estadística de Servicios)

Facultad de Ingeniería, Universidad de la República, Montevideo, Uruguay

CONTACT: artes@fing.edu.uy. 